\title{
Safety of intermediate dose of low molecular weight heparin in COVID-19 patients
}

\author{
Massimo Mattioli ${ }^{1}$ (D) Devis Benfaremo ${ }^{2} \cdot$ Mauro Mancini $^{3} \cdot$ Luciano Mucci $^{4} \cdot$ Paola Mainquà $^{5} \cdot$ Antonio Polenta $^{6}$. \\ Patrizia Maria Baldini ${ }^{4}$. Francesca Fulgenzi ${ }^{1}$. Donatella Dennetta ${ }^{7}$. Samuele Bedetta ${ }^{4}$. Lorenzo Gasperoni ${ }^{3}$. \\ Alessandro Caraffa ${ }^{3} \cdot$ Gabriele Frausini $^{4}$
}

Published online: 13 August 2020

(c) Springer Science+Business Media, LLC, part of Springer Nature 2020

\begin{abstract}
Coagulopathy represents one of the most important determinants of morbidity and mortality in coronavirus disease-19 (COVID-19). Whether standard thromboprophylaxis is sufficient or higher doses are needed, especially in severe patients, is unknown. To evaluate the safety of intermediate dose regimens of low-weight molecular heparin (LWMH) in COVID-19 patients with pneumonia, particularly in older patients. We retrospectively evaluated 105 hospitalized patients (61 M, $44 \mathrm{~F}$; mean age 73.7 years) treated with subcutaneous enoxaparin: $80 \mathrm{mg}$ /day in normal weight and mild-to-moderate impair or normal renal function; $40 \mathrm{mg} /$ day in severe chronic renal failure or low bodyweight $(<45 \mathrm{~kg}) ; 100 \mathrm{mg} /$ day if bodyweight was higher than $100 \mathrm{~kg}$. All the patients had radiologically confirmed pneumonia and $63.8 \%$ had severe COVID-19. None of the patients had fatal haemorrhage; two (1.9\%) patients had a major bleeding event (one spontaneous hematoma and one gastrointestinal bleeding). Only $6.7 \%$ of patients needed transfusions of red blood cells. One thrombotic event (pulmonary embolism) was observed. When compared to younger patients, patients older than 85 years had a higher mortality ( $40 \%$ vs 13.3\%), but not an increased risk of bleeding or need for blood transfusion. The use of an intermediate dose of LWMH appears to be feasible and data suggest safety in COVID-19 patients, although further studies are needed.
\end{abstract}

Keywords Heparin $\cdot$ COVID-19 $\cdot$ Pneumonia $\cdot$ Bleeding $\cdot$ Safety

\section{Highlights}

Massimo Mattioli

massimo.mattioli@ospedalimarchenord.it

1 UOC Pronto Soccorso e Medicina d'Urgenza, Azienda Ospedaliera “Ospedali Riuniti Marche Nord”, Piazzale Cinelli, 611214 Pesaro, Italy

2 Dipartimento di Scienze Cliniche e Molecolari, Università Politecnica delle Marche, Ancona, Italy

3 Internal Pharmacy Department, Azienda Ospedaliera "Ospedali Riuniti Marche Nord", Pesaro, Italy

4 UOC Medicina Interna, Azienda Ospedaliera "Ospedali Riuniti Marche Nord", Pesaro, Italy

5 UOC Geriatria, Azienda Ospedaliera "Ospedali Riuniti Marche Nord", Pesaro, Italy

6 UOC Malattie Infettive, Azienda Ospedaliera "Ospedali Riuniti Marche Nord", Pesaro, Italy

7 UO Pneumologia, Azienda Ospedaliera “Ospedali Riuniti Marche Nord", Pesaro, Italy
- COVID-19 coagulopathy is an emerging challenge.

- Thrombosis is one of the most relevant complications of COVID-19 patients and higher doses of heparin might be needed to prevent thrombotic events.

- Bleeding rates and mortality reported are comparable to other COVID-19 cohorts.

- In elderly patients, an intermediate dose heparin regimen is as safe as in younger patients.

- Clinical trials are urgently needed to establish safety and efficacy of anticoagulation in COVID-19.

\section{Introduction}

The importance of coagulopathy in coronavirus disease-19 (COVID-19) is emerging rapidly. The abnormal inflammatory response of the host to infection and the cytokine 
storm may play a crucial role in the endothelial dysfunction that ends up in a hypercoagulability state [1]. Autopsy studies revealed a high prevalence of thrombotic complications, mostly in pulmonary district [2]; besides, previous reports suggested heparin resistance in infected patients, due to increased factor VIII serum concentration, that could require a higher dose to be effective in thromboprophylaxis [3]. At the clinical level, in a Chinese cohort anticoagulation therapy has been associated with a better outcome in COVID-19 septic patients [4]. Therefore, anticoagulation at high doses may become the standard-of-care in the treatment of COVID-19. However, this approach may not be feasible and safe in all patients, as the risk of bleeding may be meaningful, especially in older and comorbid patients. In this study, we retrospectively analysed a cohort of hospitalized COVID-19 patients that received intermediate doses of low molecular weight heparin (LMWH) focusing on feasibility and safety.

\section{Methods}

\section{Study design}

This study follows the Strengthening the Reporting of Observational Studies in Epidemiology (STROBE) reporting guideline. This is a retrospective cohort study of consecutive patients, older than 18 years, with confirmed COVID19 infection hospitalized at Azienda Ospedaliera Ospedali Riuniti Marche Nord (Pesaro), Italy. According to hospital data, patients were admitted from March 15, 2020 to April 27, 2020. Data were collected using medical records and with a phone call for the 30-day follow-up, also used to obtain informed consent. The local ethics committee waived approval of the study given its retrospective design. Inclusion criteria were: (i) age $>18$ years; (ii) diagnosis of COVID-19 pneumonia according to the World Health Organization's interim guidance [5]. Patients discharged, transferred in Intensive Care Unit (ICU), or dead within $48 \mathrm{~h}$ after admission were excluded. Patients were defined to have severe COVID-19 if at least one of the following was present: respiratory rate $\geq 30$ breaths /min; arterial oxygen saturation $\leq 93 \%$ at rest; $\mathrm{PaO} 2 / \mathrm{FiO}_{2} \leq 300 \mathrm{mmHg}$ [6]. Major bleeding was defined by the International Society on Thrombosis and Haemostasis consensus [7]. All the other patients were considered mild-to-moderate COVID-19. The study was performed according to the ethical guidelines of the Declaration of Helsinki (seventh revision).

\section{Enoxaparin administration protocol}

The patients were treated with "intermediate" doses of LMWH, according to our Hospital Task Force for Therapy in COVID-19 recommendations, as a single daily subcutaneous injection of enoxaparin: $40 \mathrm{mg} /$ day (4000 IU) in patients with moderate-to-severe renal failure (CKD) $(15-30 \leq \mathrm{ml} /$ $\left.\mathrm{min} / 1.73 \mathrm{~m}^{2}\right)$ or low body weight $(<45 \mathrm{~kg}) ; 80 \mathrm{mg} /$ day (8000 IU) in patients with mild-to-moderate CKD (>30 ml/ $\min / 1.73 \mathrm{~m}^{2}$ ) or normal renal function and normal body weight $(45-100 \mathrm{~kg}) ; 100 \mathrm{mg} / \mathrm{day}(10,000 \mathrm{IU})$ in patients with high body weight ( $>100 \mathrm{~kg}$ ). Renal function was assessed with MDRD 4-variable Equation. The assessment of renal function and body weight was made at admission; the therapy was started during hospitalization and continued at discharge if needed by clinical decision. Thirty-days after starting LMWH, investigators made a phone call assessing vital status, complications and re-hospitalizations.

\section{Data collection and statistical analysis}

Demographic, clinical, radiological and laboratory data were collected using medical records and transferred to an electronic database. Laboratory tests were collected at admission and $7 \pm 2$ days after the beginning of LMWH. National early warning scale 2 (NEWS 2) and Charlson Comorbidity Index (CCI) were calculated at admission to estimate clinical severity and comorbidity severity. Three days after the admission, the IMPROVE bleeding risk score (IBRS) and the Padua prediction score (PPS) were calculated.

Data were summarized by means of number (\%) if categorical and median $\left(1^{\text {st }}-3^{\text {rd }}\right.$ quartiles $)$ or mean \pm SD if numerical. For the analysis, patients were stratified by age in two groups ( $<$ and $\geq 85$ years). Comparisons between the two groups were carried out by means of chi-square test or Wilcoxon-Mann-Whitney test, as appropriate. A p $<0.05$ was considered significant.

\section{Results}

Table 1 shows the baseline characteristics of 105 patients (61 M, $44 \mathrm{~F}$; mean age $73.7 \pm 14.6$ years) with COVID-19 admitted and included in the analysis (112 patients were eligible: 6 patients were excluded for missing data and 1 patient was lost at follow-up). The median duration of symptoms before admission was 8 (1-11) days. The most common symptom was fever $(77.1 \%)$, followed by dyspnea, cough and gastrointestinal symptoms $(70.4 \%, 63.8 \%$ and $11.4 \%$ of patients, respectively).

Among comorbidities, 58 (55.2\%) patients had hypertension, $13(12.3 \%)$ type 2 diabetes, $15(14.2 \%)$ were obese and $11(10.4 \%)$ were current smokers. The median CCI was 4 (3-6). On admission, COVID-19 was severe in 67 $(63.8 \%)$ patients. The majority of patients had a low-risk NEWS $2(74,70.5 \%)$, whereas only $5(4.7 \%)$ patients had a NEWS 2 score higher than 7. Patients were mostly at high 
Table 1 Baseline characteristics of COVID-19 patients included in the study

\begin{tabular}{|c|c|c|c|c|}
\hline Variable & Total $(n=105)$ & Age $<85$ years $(n=75)$ & Age $\geq 85$ years $(n=30)$ & $\mathrm{p}$ \\
\hline Age, mean $\pm S D$ & $73.7 \pm 14.6$ & $67.1 \pm 11.8$ & $90.3 \pm 3.9$ & 0.000 \\
\hline Male, $\mathrm{n}(\%)$ & $61(58)$ & $54(72)$ & $7(23.3)$ & 0.000 \\
\hline \multicolumn{5}{|l|}{ Symptoms, n (\%) } \\
\hline Fever & $81(77.1)$ & 61 & 20 & ns \\
\hline Dyspnea & $74(70.4)$ & 50 & 24 & ns \\
\hline Cough & $67(63.8)$ & 53 & 14 & 0.02 \\
\hline GI & $12(11.4)$ & 10 & 2 & ns \\
\hline $\begin{array}{l}\text { Days from symptoms onset to admission, } \\
\text { median (1st-3rd quartiles) }\end{array}$ & $8(1-11)$ & $9(5-12)$ & $1(0-9)$ & 0.001 \\
\hline \multicolumn{5}{|l|}{ Comorbidities, n (\%) } \\
\hline Hypertension & $58(55.2)$ & 40 & 18 & ns \\
\hline Diabetes & $13(12.3)$ & 8 & 5 & ns \\
\hline Obesity & $15(14.2)$ & 9 & 6 & ns \\
\hline Smoking habit & $11(10.4)$ & 10 & 1 & $\mathrm{~ns}$ \\
\hline CCI, median (1st-3rd quartiles) & $4(3-6)$ & $3(2-5)$ & $7(6-8)$ & 0.000 \\
\hline \multicolumn{5}{|l|}{ PPS, n (\%) } \\
\hline $0-3$ & $20(19.0)$ & 20 & 0 & \multirow[t]{2}{*}{0.002} \\
\hline$\geq 4$ & $85(81.0)$ & 55 & 30 & \\
\hline \multicolumn{5}{|l|}{ IBRS, n (\%) } \\
\hline $0-6$ & $97(92.4)$ & 75 & 22 & \multirow[t]{2}{*}{0.000} \\
\hline$\geq 7$ & $8(7.6)$ & 0 & 8 & \\
\hline \multicolumn{5}{|l|}{ NEWS $2, \mathrm{n}(\%)$} \\
\hline$<4$ & $74(70.4)$ & 56 & 18 & \multirow[t]{3}{*}{ ns } \\
\hline $5-6$ & $26(24.7)$ & 17 & 9 & \\
\hline$\geq 7$ & $5(4.7)$ & 2 & 3 & \\
\hline \multicolumn{5}{|l|}{ Disease severity, n (\%) } \\
\hline Mild-to-moderate & $38(36.2)$ & 25 & 13 & \multirow[t]{2}{*}{ ns } \\
\hline Severe & $67(63.8)$ & 50 & 17 & \\
\hline \multicolumn{5}{|l|}{ Creatinine clearance on admission, $\mathrm{n}(\%)$} \\
\hline$<30 \mathrm{ml} / \mathrm{min} / 1.73 \mathrm{~m}^{2}$ & $12(11.5)$ & 3 & 9 & \multirow[t]{3}{*}{0.000} \\
\hline $30-60 \mathrm{ml} / \mathrm{min} / 1.73 \mathrm{~m}^{2}$ & $21(20)$ & 14 & 7 & \\
\hline$>60 \mathrm{ml} / \mathrm{min} / 1.73 \mathrm{~m}^{2}$ & $72(68.5)$ & 58 & 14 & \\
\hline \multicolumn{5}{|c|}{ Lab tests on admission, median (1st-3rd quartiles) } \\
\hline Hemoglobin, g/dl & $13.1(11.9-14)$ & $13.4(12.2-14.4)$ & $12.3(11.1-13.4)$ & 0.03 \\
\hline Lymphocytes, $\mathrm{mm}^{3}$ & $900(600-1200)$ & $900(700-1400)$ & $750(500-1200)$ & ns \\
\hline Platelets, $\mathrm{mm}^{3}$ & $193(152-281)$ & $198(149-302)$ & $181(154-266)$ & ns \\
\hline Creatinine, mg/dl & $0.9(0.7-1.2)$ & $0.9(0.7-1.1)$ & $0.9(0.7-1.8)$ & ns \\
\hline INR & $1.3(1.2-1.4)$ & $1.3(1.2-1.4)$ & $1.2(1.2-1.3)$ & ns \\
\hline aPTT, sec & $30(28-33)$ & $30(29-33)$ & $28(27-31)$ & 0.03 \\
\hline D-dimer, ng/ml FEU & $1437(810.5-2592)$ & $1345(726-2251)$ & $2038.5(1310-3540)$ & 0.01 \\
\hline $\mathrm{CRP}, \mathrm{mg} / \mathrm{L}$ & 77 (37-144.5) & $96(46-160)$ & $45(20-100)$ & 0.003 \\
\hline
\end{tabular}

GI gastrointestinal symptoms (nausea, vomit, diarrhea), CCI charlson comorbidity index, IBRS IMPROVE bleeding risk score, $P P S$ padua prediction score, NEWS 2 national early warning scale 2, FEU fibrinogen equivalent units

thrombotic risk (PPS $\geq 4$ in $81 \%$ of patients), whereas the bleeding risk score was generally low (IBRS $<7$ in $92.4 \%$ of patients). Laboratory tests on admission showed that only $12(11.5 \%)$ patients had a creatinine clearance below
$30 \mathrm{ml} / \mathrm{min}$; coagulation status was normal both ad admission and after starting heparin.

When stratified by age, elderly ( $\geq 85$ years) patients had a more pronounced lymphopenia at admission (750 vs 900/ 
$\mathrm{mm}^{3} \mathrm{~ns}$ ), worsening at day 7 (700 vs $\left.1100 / \mathrm{mm}^{3}, \mathrm{p}=0.001\right)$; conversely, D-dimer that was significatively higher at admission (2038 vs $1345 \mathrm{ng} / \mathrm{ml} \mathrm{FEU,} \mathrm{p}=0.01)$ became similar at day 7 (1358 vs $1360 \mathrm{ng} / \mathrm{ml}$ FEU, $\mathrm{p}=\mathrm{ns}$ ).

Table 2 shows the treatment administered and the outcomes. The median duration of hospitalization was 12 (7-16) days. All the patients were treated with LMWH according to the protocol. The majority received $80 \mathrm{mg} /$ day (66 patients, $62.8 \%$ ), followed by $40 \mathrm{mg} /$ day $(35,33.4 \%$, of which 16 due to CKD). Only 4 (3.8\%) patients received $100 \mathrm{mg} /$ day of LWMH. The median duration of LMWH treatment was 13 (9-19) days. Most patients also received a combination of anti-viral and anti-inflammatory medications (Table 2). Twenty (19\%) patients were taking anti-platelet agents: three patients required blood transfusion (one of them had a major bleeding event, as discussed later) and two patients died.

After a median follow-up of 36 days, 22 (21\%) patients died, of which 19 died while admitted and 3 after discharge. When compared to younger patients, patients older than 85 years had a higher mortality, but not an increased risk of bleeding (Table 2), despite all the patients with IBRS $\geq 7$ were elderly. Overall, there were one thrombotic event and two bleeding events (1.9\%). No patient died because of fatal hemorrhage. LMWH was discontinued in 4 (3.8\%) patients due to mild to moderate thrombocytopenia, with intermediate or high probability for heparin-induced thrombocytopenia [8]. A significant decrease in hemoglobin $(\geq 2 \mathrm{~g} / \mathrm{dl})$ occurred in $21(21.2 \%)$ patients, without clinical evidence of underlying bleedings. Of these patients, 7 (6.7\%) required transfusion of packed blood cells but only the two patients with major bleedings withdrew LMWH.

\section{Discussion}

In this study, we reported the outcomes of LMWH used at intermediate prophylactic doses in COVID-19 patients, focusing on the safety of this approach in elderly patients.

All of our patients had radiologically confirmed COVID19-related pneumonia. In this population a marked microvascular thrombosis and haemorrhage linked to extensive alveolar and interstitial inflammation are the most relevant pathological features [9]. These aspects have been associated with worse outcomes especially in older patients, probably because of the reduced functional reserve of spared pulmonary parenchyma [10].

In our cohort, only one thrombotic event occurred, although the true prevalence may be underestimated since our patients were not extensively screened for deep venous thrombosis or pulmonary embolism, as probably indicated [11]. The patient who developed pulmonary embolism was a 49-year-old female, with history of deep vein thrombosis (more than 10 years before) who developed subsegmental pulmonary embolism 6 days after admission while receiving $80 \mathrm{mg}$ of enoxaparin; she was the only patient with history of venous tromboembolism. Apixaban was started and she was discharged after 13 days, without long-term oxygen needing.

Two patients had a major bleeding event. The first patient was an 87-year-old man with severe COVID-19 and treated with LMWH $40 \mathrm{mg} /$ day (due to CKD), early withdrawn for the development of moderate thrombocytopenia $\left(70 \times 10^{3}\right.$ platelet $/ \mathrm{mm}^{3}$ was the lowest count $)$; aspirin was taken as concomitant therapy. He developed spontaneous hematomas of the sternocleidomastoid muscles, bilaterally, and of the left adductor muscles, that required prolongation of the hospital stay and transfusion of three units of packed red blood cells. The patient was discharged in a post-acute care facility seven days after the onset of bleeding, without any recurrence. The second bleeding case was a 66-yearold man with severe COVID-19, who developed hematemesis for acute gastritis with active bleeding erosions. $\mathrm{He}$ was transfused (three units of packed red blood cells) and transferred to ICU, where he died 7 days later for a central venous catheter related-septic shock.

Regarding the 21 dead patients, the median age was 81.9 years (11 male) and none of them had evidence of a haemorrhagic death. Five of them had at admission, or developed during hospitalization, a D-dimer level >5000 ng/ ml FEU: all these patients performed a venous Doppler ultrasound exam of legs without evidence of thrombosis. Six of them, all younger than 71 years, was transferred in ICU.

Indeed, the use of intermediate doses of LMWH appears to be feasible and safe also in elderly patients: all the patients treated with a high-risk of bleeding (IBRS $\geq 7$ ) were older than 85 years but none of them developed major bleedings. The cumulative risk of major bleeding in our cohort was low (2\%), whereas the overall mortality (21\%) was similar to the one reported in previous studies investigating LMWH use in COVID-19 patients [12]. Unfortunately, published studies, mostly observational, reported conflicting results regarding anticoagulation treatment in COVID-19 patients. Notably, even if there are no univocal strategies in the type of heparin and administration protocols, LWMH is widely recommended in all the spectrum of disease severity, particularly in ICU patients [13-15]. Unfractionated heparin (UFH) could be used in patients with renal impairment or at high bleeding risk who need rapid reversal [16]; however, it requires expertise in the management. Some authors also suggested a possible advantage of direct oral anticoagulants in thromboprophylaxis [17]. In our cohort, anticoagulation with intermediate doses of LMWH appears to be feasible and safe also in patients with CKD. In fact, no major bleeding occurred in these patients and, additionally, the proportion of patients requiring transfusion of packed red cells was 
Table 2 Treatment and outcomes in 105 COVID-19 patients treated with LMWH

\begin{tabular}{|c|c|c|c|c|}
\hline Variable & Total $(n=105)$ & Age $<85$ years $(n=75)$ & Age $\geq 85$ years $(n=30)$ & $\mathrm{p}$ \\
\hline Duration of hospitalization (days), median ( $1^{\text {st-3rd }}$ quartiles) & $12(7-16)$ & $12(7-16)$ & $11(7-18)$ & ns \\
\hline \multicolumn{5}{|l|}{ LMWH dose, $\mathrm{n}(\%)$} \\
\hline $40 \mathrm{mg} / \mathrm{day}^{\#}$ & $35(33.4)$ & $13(17.3)$ & $22(73.3)$ & 0.000 \\
\hline $80 \mathrm{mg} /$ day & $66(62.8)$ & $58(77.3)$ & $8(26.7)$ & 0.000 \\
\hline $100 \mathrm{mg} /$ day & $4(3.8)$ & $4(5.4)$ & $0(0)$ & ns \\
\hline Duration of LMWH treatment (days), median (1st-3rd quartiles) & $13(9-19)$ & $12(9-19)$ & $13(10-23)$ & ns \\
\hline $\begin{array}{l}\text { Days from symptoms onset to LMWH start, median (1st-3rd } \\
\text { quartiles) }\end{array}$ & $8(2-12)$ & $9(1-13)$ & $1(1-9)$ & 0.009 \\
\hline \multicolumn{5}{|l|}{ Concomitant medications, n (\%) } \\
\hline Proton pump inhibitors & $81(77.1)$ & 57 & 24 & ns \\
\hline Antiplatelet agents & $20(19)$ & 13 & 7 & ns \\
\hline Corticosteroids & $54(51.4)$ & 40 & 14 & ns \\
\hline Lopinavir/ritonavir & $3(2.9)$ & 2 & 1 & ns \\
\hline Darunavir/cobicistat & $10(9.5)$ & 9 & 1 & ns \\
\hline Hydroxychloroquine & $84(80)$ & 67 & 17 & 0.000 \\
\hline Tocilizumab & $31(29.5)$ & 31 & 0 & 0.000 \\
\hline Baricitinib & $2(1.9)$ & 2 & 0 & ns \\
\hline Ruxolitinib & $1(0.9)$ & 1 & 0 & ns \\
\hline Azithromycin & $9(8.6)$ & 7 & 2 & ns \\
\hline Respiratory failure, n (\%) & $80(76.2)$ & $56(74.7)$ & $24(80)$ & ns \\
\hline \multicolumn{5}{|l|}{ On-top oxygen treatment, $\mathrm{n}(\%)$} \\
\hline Venturi mask & $45(42.8)$ & 28 & 17 & ns \\
\hline Reservoir & $12(11.4)$ & 6 & 6 & ns \\
\hline NIMV & $23(21.9)$ & 21 & 2 & 0.01 \\
\hline Duration of follow-up (days), median (1st-3rd quartiles) & $36(24-43)$ & $40(25-46)$ & $25(17-39)$ & 0.001 \\
\hline Death, n $(\%)$ & $22(21)$ & $10(13.3)$ & $12(40)$ & 0.002 \\
\hline In-hospital & 19 & 9 & 11 & \\
\hline After discharge & 3 & 1 & 2 & \\
\hline Death due to fatal hemorrhage, $\mathrm{n}(\%)$ & $0(0)$ & & & \\
\hline Admission to ICU, n (\%) & $10(9.5)$ & $10(13.3)$ & $0(0)$ & 0.03 \\
\hline \multicolumn{5}{|l|}{ Thrombotic events, $\mathrm{n}(\%)$} \\
\hline Pulmonary embolism & $1(1)$ & $1(1.33)$ & $0(0)$ & ns \\
\hline Deep venous thrombosis & $0(0)$ & & & \\
\hline Stroke & $0(0)$ & & & \\
\hline Myocardial infarction & $0(0)$ & & & \\
\hline \multicolumn{5}{|l|}{ Bleeding events, n (\%) } \\
\hline Total & $2(1.9)$ & 1 & 1 & ns \\
\hline $\begin{array}{l}\text { Major bleeding (fall in haemoglobin } \geq 2 \mathrm{~g} / \mathrm{dl} \text { or blood transfu- } \\
\text { sions } \geq 2 \text { units) }\end{array}$ & $2(1.9)$ & 1 & 1 & \\
\hline Minor bleeding & $0(0)$ & 0 & 0 & \\
\hline Thrombocytopenia, n (\%) & $4(3.8)$ & 3 & 1 & \\
\hline Loss of hemoglobin $\geq 2 \mathrm{~g} / \mathrm{dl} * *, \mathrm{n}(\%)$ & $21(21.2)$ & $16(23.2)$ & $5(17.2)$ & ns \\
\hline Blood transfusions needed, n (\%) & $7(6.7)$ & $5(6.7)$ & $2(6.7)$ & ns \\
\hline One unit & $1(0.9)$ & & & \\
\hline Two units & $4(3.8)$ & & & \\
\hline Three units & $2(1.9)$ & & & \\
\hline LMWH at discharge, $\mathrm{n}(\%)$ & $28(32.6)$ & $22(33.3)$ & $26(30)$ & ns \\
\hline \multicolumn{5}{|l|}{ Lab tests at day 7, median (1st-3rd quartiles) } \\
\hline Hemoglobin, g/dl & $12.1(10.9-13)$ & $12.2(11.2-13)$ & $11.7(10.4-12.7)$ & ns \\
\hline Lymphocytes, $\mathrm{mm}^{3}$ & $1000(500-1500)$ & $1100(700-1600)$ & $700(350-1000)$ & 0.001 \\
\hline
\end{tabular}


Table 2 (continued)

\begin{tabular}{|c|c|c|c|c|}
\hline Variable & Total $(n=105)$ & Age $<85$ years $(n=75)$ & Age $\geq 85$ years $(n=30)$ & $\mathrm{p}$ \\
\hline Platelets, $\mathrm{mm}^{3}$ & $278.5(186-348)$ & $297(217-366)$ & $217(167-316)$ & ns \\
\hline Creatinine, mg/dl & $0.8(0.6-1.1)$ & $0.75(0.6-0.9)$ & $0.85(0.6-1.6)$ & ns \\
\hline INR & $1.25(1.2-1.4)$ & $1.3(1.2-1.4)$ & $1.2(1.2-1.3)$ & ns \\
\hline aPTT, sec & $29(27-30)$ & $30(27-31)$ & $28(27-30)$ & ns \\
\hline D-dimer, ng/ml FEU & $1360(869-2294)$ & $1360(748-2263)$ & $1358.5(1024-2318.5)$ & ns \\
\hline \multicolumn{5}{|c|}{ Creatinine clearance at day $7, \mathrm{n}(\%)^{*}$} \\
\hline$<30 \mathrm{ml} / \mathrm{min} / 1,73 \mathrm{~m} 2$ & $11(11.1)$ & 1 & 10 & 0.000 \\
\hline $30-60 \mathrm{ml} / \mathrm{min} / 1,73 \mathrm{~m} 2$ & $15(15.2)$ & 9 & 6 & ns \\
\hline$>60 \mathrm{ml} / \mathrm{min} / 1,73 \mathrm{~m} 2$ & $73(73.7)$ & 59 & 14 & 0.002 \\
\hline
\end{tabular}

Bold value indicates significant at $\mathrm{p}<0.05$

$L M W H$ low molecular weight heparin, NIMV non-invasive mechanical ventilation, FEU FIbrinogen equivalent units

${ }^{\#}$ Of which 16 due to reduced creatinine clearance; *6 patients died before day 7 ; **Laboratory findings without evidence of bleedings

comparable between patients with or without CKD (5.4\% vs $16.7 \%, p=0.14)$. A relevant warning might be the decrease in hemoglobin $(\geq 2 \mathrm{~g} / \mathrm{dl})$ founded in about a fifth of patients; this could represent a hallmark of clinically overt minor bleedings. Anyway, hospital-acquired anemia (HAA) must be considered as a valid alternative explanation: large cohort studies reported prevalence of HAA between 33 and $74 \%$ in medical or critical patients hospitalized without anemia at admission [18].

Laboratory findings are consistent with literature data [19]. An interesting finding is the decrease of D-dimer level at day 7 , particularly in the elderly population. Since higher D-dimer levels have been associated with a poor prognosis, the decrease could reasonably represent a favourable effect of higher-dose heparin, although this remains to be evaluated in proper clinical trials.

Our study has several limitations, that could represent a selection bias: the monocentric cohort, the retrospective design, the small number of patients and the absence of a control group treated with standard therapy.

\section{Conclusions}

In conclusion, using intermediate doses of LMWH in COVID-19 patients is feasible and associated with a low rate of adverse events, suggesting safety, despite the absence of a control group. In the elderly and in patients with CKD, it appears to be as safe as in the other population. In addition, IBRS can be helpful in the management of COVID-19 patients at low-risk of bleedings.

Hypothetically, LMWH could represent an effective therapy directed to restore endothelial function in COVID-19 $[1,8]$. At the time of writing, a number of ongoing clinical trials are exploring this topic (EudraCT: 2020-001891-14;
EudraCT: 2020-001709-21; EudraCT: 2020-00130840; NCT04366960; NCT04373707; NCT04345848; NCT04360824; NCT04367831; NCT04359277; NCT04377997; NCT04372589; NCT04362085; NCT04344756). The results of these studies, especially of randomized controlled trials, are needed soon in order to confirm the safety and also assess the efficacy of anticoagulation treatment in COVID-19.

Author contributions MasM and GF conceived the study. MasM, MauM, LM, PM, AP, PB, FF, DD were involved in data collection. DB processed statistical analysis. MasM and DB wrote the manuscript. All the authors reviewed the draft and approved the final version of the manuscript.

Funding None.

Data availability Original data files are fully available if required to corresponding author.

\section{Compliance with ethical standards}

Conflict of interest All the authors declare that they have no conflict of interest.

Ethical approval The local ethics committee waived approval of the study given its retrospective design.

Informed consent Consent to participate: Acquired and collected. Consent for publication: All the authors gave their consent for publication.

\section{References}

1. Tay MZ, Poh CM, Rénia L, MacAry PA, Ng LFP (2020) The trinity of COVID-19: immunity, inflammation and intervention. Nat Rev Immunol. https://doi.org/10.1038/s41577-020-0311-8 
2. Wichmann D, Sperhake J, Lütgehetmann M et al (2020) Autopsy findings and venous thromboembolism in patients with COVID19: a prospective cohort study. Ann Intern Med. https://doi. org/10.7326/M20-2003

3. Beun R, Kusadasi N, Sikma M, Westerink J, Huisman A (2020) Thromboembolic events and apparent heparin resistance in patients infected with SARS-CoV-2. Int J Lab Hematol. https:// doi.org/10.1111/ijlh.13230

4. Tang N, Bai H, Chen X, Gong J, Li D, Sun Z (2020) Anticoagulant treatment is associated with decreased mortality in severe coronavirus disease 2019 patients with coagulopathy. J Thromb Haemost 18:1094-1099. https://doi.org/10.1111/jth.14817

5. World Health Organization (2020) Clinical management of severe acute respiratory infection when novel coronavirus $(\mathrm{nCoV})$ infection is suspected: interim guidance. https://www.who.int/publi cations-detail/clinical-management-of-severeacute-respirator $\mathrm{y}$-infection-when-novelcoronavirus-(ncov)-infection-is-suspe ctedAccessed 20 May 2020

6. Berlin DA, Gulick RM, Martinez FJ (2020) Severe Covid-19. N Engl J Med. https://doi.org/10.1056/NEJMcp2009575

7. Schulman S, Kearon C (2005) Subcommittee on Control of Anticoagulation of the Scientific and Standardization Committee of the International Society on Thrombosis and Haemostasis. Definition of major bleeding in clinical investigations of antihemostatic medicinal products in non-surgical patients. J Thromb Haemost 3(4):692-694. https://doi.org/10.1111/j.1538-7836.2005.01204.x

8. Lo GK, Juhl D, Warkentin TE, Sigouin CS, Eichler P, Greinacher A (2006) Evaluation of pretest clinical score (4 T's) for the diagnosis of heparin-induced thrombocytopenia in two clinical settings. J Thromb Haemost 4(4):759-765. https://doi.org/10.11 11/j.1538-7836.2006.01787.x

9. McGonagle D, O'Donnell JS, Sharif K, et al (2020) Immune mechanisms of pulmonary intravascular coagulopathy in COVID19 pneumonia. Lancet Rheum. https://doi.org/10.1016/S2665 -9913(20)30121-1

10. Chen N, Zhou M, Dong X et al (2020) Epidemiological and clinical characteristics of 99 cases of 2019 novel coronavirus pneumonia in Wuhan, China: a descriptive study. Lancet 395:507-513
11. Lodigiani C, Iapichino G, Carenzo L et al (2020) Venous and arterial thromboembolic complications in COVID-19 patients admitted to an academic hospital in Milan. Italy Thromb Res 191:9-14. https://doi.org/10.1016/j.thromres.2020.04.024

12. Paranjpe I, Fuster V, Lala A et al (2020) Association of treatment dose anticoagulation with in-hospital survival among hospitalized patients with COVID-19. J Am Coll Cardiol. https://doi. org/10.1016/j.jacc.2020.05.001

13. Casini A, Alberio L, Angelillo-Scherrer A et al (2020) Thromboprophylaxis and laboratory monitoring for in-hospital patients with COVID-19: a Swiss consensus statement by the Working Party Hemostasis. Swiss Med Wkly. https://doi.org/10.4414/ smw.2020.20247

14. Connors JM, Levy JH (2020) COVID-19 and its implications for thrombosis and anticoagulation. Blood. https://doi.org/10.1182/ blood 2020006000

15. Bikdeli B, Madhavan MV, Jimenez D et al (2020) COVID-19 and thrombotic or thromboembolic disease: implications for prevention, antithrombotic therapy, and follow-up. J Am Coll Cardiol. https://doi.org/10.1016/j.jacc.2020.04.031

16. Thachil J, Tang N, Gando S et al (2020) Type and dose of heparin in Covid-19: Reply. J Thromb Haemost. https://doi.org/10.1111/ jth. 14870

17. Spyropoulos AC, Ageno W, Barnathan ES (2020) Hospital-based use of thromboprophylaxis in patients with COVID-19. Lancet 395(10234):e75. https://doi.org/10.1016/S0140-6736(20)30926-0

18. Shander A, Corwin HL (2020) A narrative review on hospitalacquired anemia: keeping blood where it belongs. Transfus Med Rev. https://doi.org/10.1016/j.tmrv.2020.03.003

19. Terpos E, Ntanasis-Stathopoulos I, Elalamy I et al (2020) Hematological findings and complications of COVID-19. Am J Hematol. https://doi.org/10.1002/ajh.25829

Publisher's Note Springer Nature remains neutral with regard to jurisdictional claims in published maps and institutional affiliations. 